\title{
Evidências de Validade Convergente-Discriminante para a Avaliação dos Tipos Profissionais de Holland (ATPH)
}

Convergent-Discriminant Validity Evidences For The Avaliation Of Holland's Professional Types (ATPH)

Evidencias De Validez Convergente-Discriminante Para La Evaluación De Los Tipos Profesionales De Holland (ATPH)

Ana Paula Porto Noronha, Camélia Santina Murgo Mansão, Marlene Alves da

Silva, Patrícia Carla dos

Santos Freitas \& Geruza

Oliveira Aquino Pereira

Universidade São Francisco

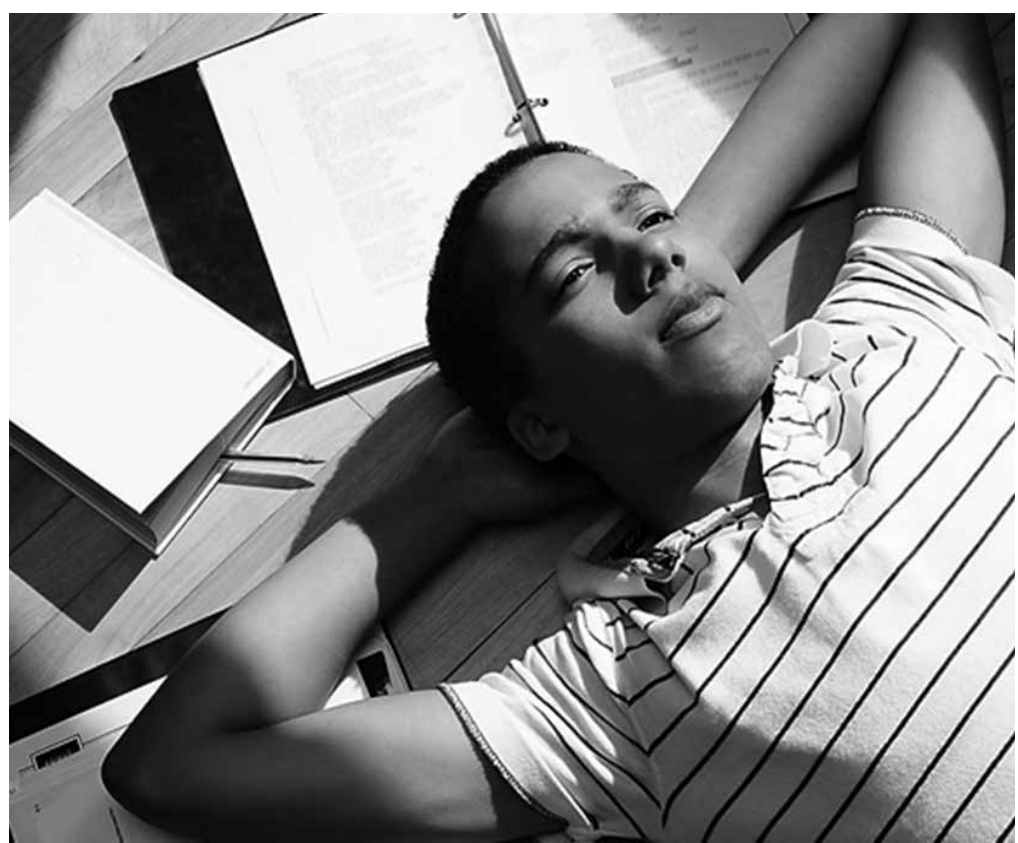


Resumo: Este estudo teve como objetivo buscar evidências de validade convergente-discriminante para o teste Avaliação dos Tipos Profissionais, de Holland (ATPH), com vistas a verificar as relações com a Escala de Aconselhamento Profissional (EAP). A amostra foi composta por 42 estudantes dos ensinos fundamental e médio de escolas públicas e particulares. A idade variou entre 11 e 26 anos, sendo que participaram $43 \%$ de alunos do sexo masculino, 50\%, do feminino, e $7 \%$ que não informaram o gênero. Os resultados sugeriram correlações significativas entre as dimensões do EAP e as tipologias do ATPH, com magnitudes que variavam de baixas a moderadas. A partir dos dados coletados, concluiu-se que o ATPH pode ajudar na identificação dos interesses de estudantes em processos de orientação profissional e que as evidências de validade foram favoráveis.

Palavras-chave: Interesses. Orientação vocacional. Avaliação psicológica. Adolescentes.

Abstract: The present study aimed to search convergent-discriminant validity evidences for Holland's test Avaliação dos Tipos Profissionais (ATPH) by verifying its relations with the Escala de Aconselhamento Profissional (EAP). The sample was composed by 42 elementary school and high school students, from public and private schools. The age ranged from 11 to 26 years old, with $43 \%$ men, $50 \%$ women and $7 \%$ that didn't mention their gender. The results suggested significant correlations between EAP dimensions and ATPH typologies, ranging from low to moderated magnitudes. From the collected data, it's possible to conclude that ATPH can help to identify the interests of the students that undergo career counseling and that its validity evidences were favorable.

Keywords: Interests. Vocational guidance. Psychological assesment. Adolescents.

Resumen: Este estudio tuvo como objetivo buscar evidencias de validez convergente-discriminante para la prueba Evaluación de los Tipos Profesionales, de Holland (ATPH), con vistas de verificar las relaciones con la Escala de Consejería Profesional (EAP). La muestra estuvo compuesta de 42 estudiantes de los ciclos primario y secundario de escuelas públicas y particulares. La edad varió entre 11 y 26 años, y participaron $43 \%$ de alumnos del sexo masculino, $50 \%$, del femenino, y $7 \%$ que no informaron el género. Los resultados sugirieron correlaciones significativas entre las dimensiones del EAP y las tipologías del ATPH, con magnitudes que variaban de bajas a moderadas. A partir de los datos recolectados, se concluyó que el ATPH puede ayudar en la identificación de los intereses de estudiantes en procesos de orientación profesional y que las evidencias de validez fueron favorables.

Palabras clave: Intereses. Orientación vocacional. Evaluación psicológica. Adolescentes.

O construto interesse tem recebido atenção no âmbito da Psicologia, em especial na orientação profissional (Holland, 1975; Mansão \& Yoshida, 2008; Noronha, Martins, Gurgel \& Ambiel, 2009; Sartori, Noronha, \& Nunes, 2009; Savickas, 1995; Super, 1953). Para Crites (1969), os interesses são construídos ao longo do desenvolvimento vocacional, que, por sua vez, sofre influência de variáveis inter-relacionadas. Estas são denominadas, pelo autor, de variáveis de estímulo (incluem a cultura, a classe social, a região geográfica, a comunidade, a família e a escola), variáveis orgânicas (incluem o funcionamento biológico, o psicológico e as características hereditárias), e variáveis de resposta (incluem atitudes de modo geral e as características de personalidade).

No que se refere à classificação dos interesses, para Super (1953), os pesquisadores criam nomenclaturas conforme os processos empregados e as pessoas estudadas. Como é comum acontecer em situações de pesquisa, as descobertas são limitadas pela natureza dos dados com que se trabalha e pelo enfoque teórico utilizado. Grande parte dos estudos sobre interesses profissionais na Psicologia foi feita por psicólogos americanos que possuíam sólida orientação experimental. Assim sendo, as teorias surgiram a partir da interpretação de dados empiricamente coletados.

Ainda no que se refere às tentativas de conceituação, outros autores, mais recentemente, trouxeram suas contribuições. Savickas (1995), por exemplo, afirmou que é possível atribuir ao termo interesse múltiplos significados. Em um sentido mais amplo, refere-se à posição de um indivíduo em relação a um objeto específico, em termos da atenção que desperta no sujeito, 
Para Holland

(1975), tipo é um modelo que serve para medir a pessoa real.

Cada tipo é uma interação entre uma herança determinada e uma variedade de fatores culturais e pessoais, que incluem as outras pessoas semelhantes, os pais, os adultos significativos, a cultura e 0 ambiente físico. do sentimento do indivíduo em relação ao objeto que acompanha a atenção (prazeroso/ desprazeroso), e da tendência a aproximarse ou não do objeto (querer ou não interagir com ele). Já quando o interesse é empregado especialmente no âmbito profissional, pode designar grupos de interesses específicos, razoavelmente homogêneos e relacionados entre si, que constituem, então, uma disposição que tende a ser mais estável. Sob essa perspectiva, eles devem ser entendidos mais como traço do que como estado. Assim, os interesses denotam uma tendência de resposta razoavelmente estável, consistente e persistente, que aumenta a prontidão de um indivíduo para atender a certos tipos de estímulos ambientais e para agir em relação a eles.

A Escala de Aconselhamento Profissional (EAP), um dos instrumentos utilizados neste estudo, verifica interesses profissionais a partir de itens descritores de atividades ocupacionais que contemplam sete dimensões: ciências exatas, artes e comunicação, ciências biológicas e da saúde, ciências agrárias e ambientais, atividades burocráticas, ciências humanas e sociais aplicadas e entretenimento. A escala foi elaborada em comunhão com as asserções de Savickas (1995), no sentido de que interesses podem ser definidos como gosto/ desgosto (do inglês like/dislike) em relação a determinadas atividades profissionais.

Ainda no que diz respeito às perspectivas teóricas que explicam os interesses, também se pode discutir a tipologia de Holland (1975), uma vez que o instrumento para o qual se busca evidências de validade no presente estudo (ATPH) foi construído com base nesse arcabouço teórico. Holland propôs um instrumento de avaliação intitulado selfdirected search career explorer (SDS), que se apoia em uma tipologia profissional composta por seis tipos de personalidade e seis modelos ambientais. Tem-se, portanto: realista, investigativo, artístico, social, empreendedor, convencional e, igualmente, os modelos ambientais, nomeados tal como os anteriores.

Para Holland (1975), tipo é um modelo que serve para medir a pessoa real. Cada tipo é uma interação entre uma herança determinada e uma variedade de fatores culturais e pessoais, que incluem as outras pessoas semelhantes, os pais, os adultos significativos, a cultura e o ambiente físico. As heranças biológica e social, unidas à história pessoal, auxiliam na formação de capacidades, de habilidades perceptivas e de pontos de vista como metas, valores, autoconceito e condutas de enfrentamento (estratégias específicas para resolver os problemas cotidianos), portanto, um tipo é uma completa síntese de características pessoais.

O tipo realista refere-se ao masculino, fisicamente forte, pouco sociável; tem boa coordenação motora e rapidez e prefere os problemas concretos aos abstratos. $\mathrm{O}$ tipo investigativo é hábil em lidar com a manipulação de palavras e ideias, e, ao lado disso, é analítico, introvertido e crítico, e possui valores pouco convencionais e uma necessidade permanente de compreender as coisas. O tipo social tende a ser responsável, sensível, humanista. As pessoas desse tipo têm muita necessidade de interação social e costumam confiar mais nos sentimentos do que no lado racional para a solução de problemas. O tipo convencional mostrase eficiente em tarefas bem organizadas, identifica-se com o poder, valoriza os bens materiais e a posição social e está atento às metas, aos conceitos e aos valores aceitos pela sociedade; demonstra-se inflexível, rígido e carente de criatividade. O tipo empreendedor é caracterizado como aventureiro, dominante, entusiasta, impulsivo e extrovertido, com boa capacidade verbal para vender, dominar e persuadir, gosta de assuntos ligados à política e à economia, e mostra preocupação com o poder, a posição social e a liderança. Por fim, 
o tipo artístico costuma enfrentar as situações do cotidiano utilizando-se dos sentimentos, das emoções, das intuições e da imaginação.

A partir das tipologias que descreveu, o autor criou o SDS - Questionário de Busca Autodirigida, que consiste em um inventário de interesses que se baseia no modelo hexagonal e que vem sofrendo repetidas revisões desde sua primeira publicação. Além da forma regular (forma $R$ ), existem três outras versões: a forma E - easy - aplicável em indivíduos com pouca habilidade de leitura, a forma CP - career planning - utilizada com adultos em processo de reorientação de carreira, e a forma CE - career explorer (Holland, Fritzshe, \& Powell, 1994).

O SDS tem um grande escopo de pesquisas desenvolvidas, enquanto o EAP, por ser um instrumento relativamente novo, ainda carece de novas investigações. Alguns resultados de ambos os instrumentos serão apresentados a seguir. Sartori, Noronha e Nunes analisaram as diferenças de médias entre o EAP e o SDS em uma amostra de 177 estudantes do ensino médio de escolas do Estado de São Paulo. Em relação ao EAP, foram observadas diferenças para as dimensões ciências exatas (médias maiores para homens) e ciências biológicas e da saúde e ciências agrárias e ambientais (médias maiores para mulheres).

Com a intenção de verificar a forma de interpretação das dimensões do EAP, Sisto, Santos e Noronha (2009) aplicaram a escala em 762 estudantes universitários. Dentre os principais resultados, serão destacados os que mais se coadunam com este estudo. $\mathrm{Na}$ dimensão ciências exatas, o maior destaque foi para o curso de Engenharia, ao passo que Educação Física, Turismo, Pedagogia e Jornalismo se destacaram na dimensão artes, e Comunicação, Medicina, Fisioterapia e Veterinária tiveram as maiores médias das ciências biológicas e da saúde, Turismo e Veterinária tiveram as maiores médias das ciências agrárias e ambientais, e Administração e Direito, nas atividades burocráticas. As ciências humanas e sociais aplicadas incluíram os cursos de Pedagogia, Jornalismo, Psicologia e Direito, e, por fim, o de Turismo teve maior média no entretenimento.

Noronha e Ambiel (2008) buscaram a correlação entre os itens da Escala de Aconselhamento Profissional (EAP) com os itens da escala Atividades do Self-Directed Search (SDS) em uma amostra formada por 122 estudantes de Psicologia, Educação Artística e Veterinária. Na análise dos resultados, pôdese perceber que os estudantes de Educação Artística apresentaram médias mais elevadas nos tipos artístico e social do SDS; os de Psicologia, nos tipos social e investigativo, e os de Veterinária, nos tipos empreendedor e investigativo. Na média geral, os tipos social e artístico foram predominantes. No que se refere aos resultados na EAP, Veterinária obteve a maior média. Os itens da escala Atividades Profissionais foram correlacionados com os tipos preconizados pelo SDS, e, nesse sentido, para o tipo realista, o maior índice foi o item 56, que evoca a visão econômica do sujeito. As atividades voltadas para os cuidados com seres humanos foram as que se destacaram nas correlações com o tipo investigativo.

No que diz respeito à análise dos dados referentes ao tipo artístico, seis itens da EAP se correlacionaram positivamente com a Escala de Atividades do SDS, com coeficientes superiores a 0,40. Quanto ao tipo social, notou-se que os itens da EAP que se correlacionaram mais positivamente às atividades propostas pelo SDS estavam mais voltados para o estudo da origem e da evolução da História e da cultura humana e para o contato com a arte, porém em funções de coordenação de pessoal, e não em atividades artísticas puras, criativas e/ou centradas. 
Primi, Pellegrini, Nucci, Munhoz, Bighetti e Moggi (2001) correlacionaram o SDS com o IDDP - Inventário de Dificuldades de Decisão Profissional - e o IFP - Inventário Fatorial de Personalidade, baseados na expectativa de que as escalas que indicam dificuldades de escolha se correlacionariam negativamente com os demais instrumentos utilizados. Os autores aventaram a hipótese de que, quanto maior a dificuldade relatada, menor seria a diferenciação dos perfis de personalidade e de interesse. Os resultados revelaram uma única associação significativa entre a imaturidade avaliada pelo IDDP e a indiferenciação de perfis de interesses do SDS.

Quanto às subescalas do IDDP e aos tipos do modelo de Holland, obteve-se associação entre a subescala que avalia o conflito resultante da preferência por várias opções, com todos os tipos de Holland, o que foi interpretado como uma evidência de validade, uma vez que escores mais altos nessa subescala estariam associados a interesses por todos os tipos do SDS. Ainda de acordo com os resultados, alunos que relataram dificuldades econômicas preferiram os tipos realista, social, empreendedor e convencional; já os que tinham maior motivação econômica também tenderam a escores mais altos no tipo empreendedor. Por sua vez, a tendência à insegurança e à percepção de falta de informação associou-se de modo significativo ao tipo convencional (Primi et al., 2001).

Posteriormente, Primi, Moggi e Casellato (2004) estudaram o IFP e o SDS. O tipo investigativo esteve mais associado à valorização da dimensão intelectual (IFP); da mesma maneira, o tipo social relacionouse ao aspecto da cooperação social e da preocupação em não entrar em desacordo com os outros. No tipo artístico, a associação predominante foi com o fator deferência, o que pode ser entendido como tendência à admiração de pessoas que tiveram grandes feitos, ou seja, pode refletir uma necessidade de expressão que leva à admiração de pessoas grandiosas. O tipo empreendedor apareceu ligado à liderança e às atitudes executivas e competitivas, enquanto o tipo convencional, à sistematicidade. Os coeficientes de consistência interna para os tipos do modelo RIASEC variaram de 0,86 a 0,93, o que indica uma precisão muito satisfatória apesar da pequena amostra do estudo (81 participantes).

Mais recentemente, Mansão e Yoshida (2008) realizaram estudo que teve por finalidade buscar evidência de validade da versão em português do Self-Directed Search Career Explorer - SDS, a partir da versão adaptada por Primi et al., (2001). Quanto à medida de consistência interna, os resultados apontaram a homogeneidade dos itens relativos a cada um dos tipos do modelo RIASEC, seja quando se considerou o tipo de escola (particular ou pública), seja quando a amostra foi dividida segundo o sexo.

Quanto ao sistema de ensino, os coeficientes alfa encontrados variaram entre 0,88 e 0,90 para todas as tipologias, tanto no grupo dos estudantes de escolas públicas quanto nos da escola particular. Em relação ao sexo, os coeficientes variaram entre 0,82 e 0,90 tanto para o feminino quanto para o masculino. Essa constatação se aplica a todas as tipologias do SDS, ou seja, o instrumento revelou alta consistência interna. Além disso, os índices obtidos ficaram muito próximos dos reportados por Primi et al., (2004), o que reforça a interpretação de precisão satisfatória do instrumento.

A análise fatorial forneceu dados da dimensionalidade do construto, reproduzindo os componentes preconizados pelo modelo teórico. Efetivamente, os seis fatores corroboraram o pressuposto de 
que o construto interesse profissional é representado por seis tipos conforme a versão original do instrumento: realista, investigativo, artístico, social, empreendedor e convencional. Além disso, cada um deles contém itens representativos das quatro seções do instrumento (igualmente propostas na versão original), que são atividades, competências, carreiras e habilidades. A autora concluiu que, a partir do SDS, é possível avaliar interesses profissionais de forma simples e rápida, sendo sua utilização apropriada para situações individuais ou coletivas em processos de orientação profissional, nos contextos clínico ou escolar.

Com vistas a investigar as associações entre interesses e personalidade, Savikas (1999) investigou 394 trabalhadores adultos. Os instrumentos aplicados foram SDS e os cinco grandes fatores de personalidade, tendo sido encontrados padrões diferenciados de associações entre os construtos para homens e mulheres. Os fatores de abertura, extroversão e socialização facilitaram mais a diferenciação de tipos entre as mulheres, ao passo que, entre os homens, os fatores foram apenas os de abertura e extroversão.

Nauta (2007) investigou aspectos de carreira entre universitários. Participaram 115 sujeitos, e os resultados apontaram correlações significativas entre interesses e personalidade. Os instrumentos utilizados estavam baseados nos modelos de Holland e dos cinco grandes fatores de personalidade. O tipo realista relacionou-se positivamente com o fator abertura, e negativamente com o fator neuroticismo. O tipo investigativo não apresentou correlações com quaisquer traços de personalidade, e o tipo artístico correlacionou-se significativamente com abertura, socialização e neuroticismo, enquanto o tipo social, com socialização e extroversão. Por fim, houve índices significativos entre o tipo empreendedor e extroversão, e convencional e neuroticismo. No Brasil, estudos empíricos no campo da orientação profissional, mais especificamente aqueles que se referem aos instrumentos de verificação de interesses, ainda são incipientes. Considerando tal escassez de instrumentos validados na área, esta pesquisa teve como objetivos buscar evidências de validade convergente-discriminante para o ATPH - Avaliação dos Tipos Profissionais, de Holland, construído a partir das dimensões de interesses vocacionais do modelo RIASEC, por meio da comparação com a EAP - Escala de Aconselhamento Profissional.

\section{Método}

\section{Participantes}

A amostra foi composta por 42 participantes dos ensinos fundamental e médio, sendo dois estudantes do $7^{\circ}$ ano do ensino fundamental, um do $8^{\circ}$ ano e quatro do $9^{\circ}$ ano. Entre os estudantes de ensino médio, seis cursavam a primeira série, 12, a segunda, e 15, a terceira. Um participante já havia concluído o ensino médio e um não informou a escolaridade. Observou-se maior concentração entre as primeiras e segundas séries do ensino médio, com 64,5\%. Quanto aos sistemas de ensino, 38 (90,5\%) eram estudantes de escolas públicas e três (7\%) de particulares, e suas idades variaram de 11 a 26 anos, com a média de 17. Quanto ao sexo, 18 (43\%) eram homens e 21 (50\%), mulheres. Três participantes não informaram a que gênero pertenciam.

\section{Instrumentos}

O ATPH - Avaliação dos Tipos Profissionais de Holland - (Primi, Muniz, Nunes, \& 
Murgo, 2008) foi elaborado a partir do SDS - Self Directed Search - de Holland (1975), e tem como objetivo verificar interesses e preferências para elaborar um perfil tipológico. É composto por 154 itens que representam as seis tipologias de Holland: realista, intelectual, artístico, social, convencional e empreendedor. As respostas mostram a intensidade do interesse do sujeito pelas atividades profissionais descritas nos itens, e cada item é avaliado por uma escala de quatro pontos, do tipo Likert, indo de nenhum interesse (resposta 1) a muito interesse (resposta 4).

A Escala de Aconselhamento Profissional EAP (Noronha, Sisto, \& Santos, 2007) é o instrumento que avalia as preferências pelas atividades profissionais de jovens acima de 17 anos. O examinando deve quantificar com que intensidade gostaria de realizar a atividade em questão. Para a correção, devem ser somados os valores atribuídos aos itens que compõem cada dimensão avaliada. A escala é composta por 61 itens, descritores de atividades profissionais que contemplam 7 dimensões: cências exatas, artes e comunicação, ciências biológicas e da saúde, ciências agrárias e ambientais, atividades burocráticas, ciências humanas e sociais aplicadas e entretenimento.

No principal estudo das características psicométricas, com vistas a obter dados de validade e precisão, foram analisados os protocolos de 762 estudantes, com idades que variaram ente 17 e 73 anos. Os valores de alfa ficaram entre 0,79 e 0,94 , os de Spearman-Brown e Guttman, entre 0,75-0,91, valores esses que podem ser considerados altos. Com base nesses resultados, pode-se considerar que as diversas dimensões do instrumento apresentaram ótima precisão.

\section{Procedimentos}

O projeto foi submetido ao Comitê de Ética em Pesquisa e obteve a aprovação. Posteriormente, foram realizadas duas coletas para aplicação dos instrumentos, ocasião em que os aplicadores esclareceram a natureza da pesquisa e solicitaram que os jovens assinassem o Termo de Consentimento Livre e Esclarecido como requisito para participação. No caso de estudantes menores de idade, foi requerida também a autorização dos pais para participar da pesquisa. Os instrumentos foram aplicados individualmente, e todos os participantes responderam a ambos.

\section{Resultados e discussões}

Inicialmente, serão apresentadas as estatísticas descritivas das dimensões dos instrumentos. Nota-se que, no EAP, as dimensões atividades burocráticas e ciências humanas e sociais aplicadas alcançaram as maiores médias, seguidas da dimensão ciências agrárias e ambientais. Já a dimensão ciências exatas obteve a menor preferência de atividade profissional para esta amostra. Pode-se compreender que as maiores preferências dos jovens consultados são aquelas em que a organização é possível, sendo que, a título de exemplo, pode-se citar os processos de departamento de pessoal (relação empresafuncionário) e financeiro (arrecadação de impostos e taxas).

Ao lado disso, os participantes parecem gostar de livros e textos, de ler e escrever. Tendem a identificar-se com a elaboração de programas assistenciais voltados para o desenvolvimento humano, nos âmbitos sociais, educacionais e nas relações pessoaempresa. Por fim, também demonstraram interesse por atividades relativas ao meio ambiente. A Tabela 1 apresenta essas informações. 
Tabela 1. Estatísticas descritivas dos instrumentos EAP e ATPH

\begin{tabular}{llllll}
\hline EAP & $\mathrm{N}$ & Mínimo & Máximo & Média & DP \\
\hline Ciências exatas & 42 & 1,00 & 4,36 & 2,10 & 0,92 \\
Artes e comunicação & 42 & 1,14 & 4,29 & 2,32 & 0,92 \\
Ciências biológicas e da saúde & 42 & 1,00 & 4,89 & 2,27 & 1,13 \\
Ciências agrárias e ambientais & 42 & 1,00 & 4,92 & 2,34 & 1,11 \\
Atividades burocráticas & 42 & 1,00 & 4,31 & 2,36 & 0,93 \\
Ciências humanas sociais aplic. & 42 & 1,22 & 3,90 & 2,35 & 0,83 \\
Entretenimento & 42 & 1,00 & 4,50 & 2,24 & 1,05 \\
ATPH & & & & & \\
Artístico & 34 & 33 & 109,00 & 56,97 & 20,04 \\
Convencional & 35 & 23 & 76,00 & 44,63 & 16,81 \\
Empreendedor & 41 & 28 & 103,00 & 64,05 & 18,09 \\
Investigativo & 35 & 23 & 83,00 & 44,54 & 15,53 \\
Realista & 37 & 27 & 84,00 & 45,24 & 16,94 \\
Social & 37 & 25 & 96,00 & 48,70 & 18,89 \\
\hline
\end{tabular}

Com relação ao ATPH, as maiores médias foram nas tipologias empreendedor e artístico, enquanto a tipologia investigativa obteve a menor média. Assim, os jovens deste estudo se percebem como aventureiros, dominantes, entusiastas, impulsivos e extrovertidos, além de possuírem boa capacidade verbal para vender, dominar e persuadir. Também são mais dispostos a fazer uso dos sentimentos, emoções, intuições e imaginação. A seu turno, o menor interesse foi em relação à habilidade para lidar com a manipulação de palavras e de ideias.

As diferenças de média entre sexos foram analisadas e podem ser visualizadas na Tabela 2. Com relação à EAP, verificou-se que não houve diferença significativa entre os sexos em nenhuma das dimensões.

Tabela 2. Diferença de médias e teste $t$ entre sexos

\begin{tabular}{|c|c|c|c|c|c|c|}
\hline EAP & Sexo & $\mathrm{N}$ & Média & DP & $t$ & $p$ \\
\hline \multirow[t]{2}{*}{ Ciências exatas } & $M$ & 18 & 0,77 & 0,18 & 0,11 & 0,275 \\
\hline & $\mathrm{F}$ & 21 & 1,95 & 1,07 & & \\
\hline \multirow[t]{2}{*}{ Artes e comunicação } & M & 18 & 2,07 & 0,78 & 0,20 & 0,183 \\
\hline & $\mathrm{F}$ & 21 & 2,46 & 0,95 & & \\
\hline \multirow[t]{2}{*}{ Ciências biológicas e da saúde } & M & 18 & 2,06 & 1,03 & 0,37 & 0,313 \\
\hline & $\mathrm{F}$ & 21 & 2,44 & 1,25 & & \\
\hline \multirow[t]{2}{*}{ Ciências agrárias e ambientais } & M & 18 & 2,26 & 1,11 & 0,95 & 0,604 \\
\hline & $\mathrm{F}$ & 21 & 2,45 & 1,18 & & \\
\hline \multirow[t]{2}{*}{ Atividades burocráticas } & M & 18 & 2,40 & 0,78 & 0,19 & 0,620 \\
\hline & $\mathrm{F}$ & 21 & 2,25 & 1,07 & & \\
\hline \multirow[t]{2}{*}{ Ciências humanas sociais aplic. } & M & 18 & 2,18 & 0,71 & 0,08 & 0,396 \\
\hline & $\mathrm{F}$ & 21 & 2,40 & 0,90 & & \\
\hline \multirow[t]{2}{*}{ Entretenimento } & M & 18 & 2,00 & 0,99 & 0,32 & 0,282 \\
\hline & $\mathrm{F}$ & 21 & 2,37 & 1,10 & & \\
\hline \multicolumn{7}{|l|}{ ATPH } \\
\hline \multirow[t]{2}{*}{ Artístico } & M & 13 & 53,53 & 13,83 & $-0,76$ & 0,454 \\
\hline & $\mathrm{F}$ & 18 & 58,78 & 21,90 & & \\
\hline \multirow[t]{2}{*}{ Convencional } & M & 14 & 44,50 & 15,37 & 0,27 & 0,793 \\
\hline & $\mathrm{F}$ & 18 & 42,89 & 18,26 & & \\
\hline \multirow[t]{2}{*}{ Empreendedor } & M & 18 & 60,33 & 16,26 & $-0,65$ & 0,519 \\
\hline & $\mathrm{F}$ & 20 & 64,00 & 18,20 & & \\
\hline \multirow[t]{2}{*}{ Investigativo } & M & 15 & 41,67 & 15,65 & $-0,90$ & 0,376 \\
\hline & $\mathrm{F}$ & 17 & 46,82 & 16,69 & & \\
\hline \multirow[t]{2}{*}{ Realista } & M & 17 & 52,65 & 19,02 & 3,49 & 0,001 \\
\hline & $\mathrm{F}$ & 17 & 35,29 & 7,64 & & \\
\hline \multirow[t]{2}{*}{ Social } & M & 15 & 41,60 & 14,49 & $-2,05$ & 0,049 \\
\hline & $\mathrm{F}$ & 19 & 54,63 & 20,99 & & \\
\hline
\end{tabular}


Já nas dimensões do ATPH, as mulheres obtiveram médias mais altas no tipo social. Os resultados corroboram os achados de Savikas (1995), uma vez que o autor encontrou resultados maiores nas mulheres em relação às características de socialização. Diferentemente, na dimensão realista, os homens obtiveram médias mais altas; portanto, pode-se depreender que as mulheres são mais sensíveis e voltadas para os relacionamentos interpessoais, enquanto os homens são mais concretos e menos sociáveis (Holland, 1975).

Procedeu-se à correlação de Pearson, a fim de compreender a comunalidade entre os construtos dos instrumentos. Os dados são apresentados na Tabela 3.

Tabela 3. Correlações entre as dimensões da EAP e ATPH

\begin{tabular}{llllllll}
\hline & & $\mathrm{A}$ & $\mathrm{C}$ & $\mathrm{E}$ & $\mathrm{I}$ & $\mathrm{R}$ & $\mathrm{S}$ \\
\hline Ciências exatas & $\mathrm{r}$ & 0,14 & 0,37 & 0,22 & 0,40 & ${ }^{*} 0,45$ & 0,04 \\
& $\mathrm{p}$ & 0,45 & 0,31 & 0,17 & 0,02 & 0,01 & ${ }^{*} 0,84$ \\
Artes e comunicação & $\mathrm{r}$ & $* 0,60$ & 0,09 & 0,29 & 0,12 & 0,09 & 0,35 \\
& $\mathrm{p}$ & 0,00 & ${ }^{*} 0,60$ & 0,07 & 0,51 & ${ }^{*} 0,59$ & 0,03 \\
Ciências biológicas e & $\mathrm{r}$ & 0,19 & 0,18 & 0,23 & ${ }^{*} 0,62$ & 0,05 & 0,50 \\
da Saúde & $\mathrm{p}$ & 0,29 & 0,33 & 0,15 & 0,00 & $* 0,78$ & 0,00 \\
Ciências agrárias e & $\mathrm{r}$ & 0,09 & 0,12 & 0,06 & $* 0,55$ & 0,07 & 0,19 \\
Ambientais & $\mathrm{p}$ & 0,62 & 0,50 & $* 0,70$ & 0,00 & 0,68 & 0,26 \\
Atividades Burocráticas & $\mathrm{r}$ & 0,06 & $* 0,67$ & 0,45 & 0,41 & 0,40 & 0,05 \\
& $\mathrm{p}$ & 0,74 & 0,00 & 0,00 & 0,01 & 0,02 & $* 0,79$ \\
Ciências humanas e Sociais & $\mathrm{r}$ & $* 0,48$ & 0,45 & 0,47 & 0,36 & 0,15 & 0,44 \\
& $\mathrm{p}$ & 0,01 & 0,01 & 0,00 & 0,03 & $* 0,39$ & 0,01 \\
entretenimento & $\mathrm{r}$ & 0,30 & 0,37 & $* 0,44$ & 0,07 & 0,09 & 0,32 \\
& $\mathrm{p}$ & 0,09 & 0,03 & 0,00 & $* 0,67$ & 0,61 & 0,06 \\
\hline
\end{tabular}

A (artístico) C (convencional) E (empreendedor) I (investigativo) R (realista) S (social)

Foram observadas correlações significativas entre as dimensões do EAP e as tipologias do SDS, com magnitudes que variavam de baixas a moderadas. Verificou-se que as tipologias realista e investigativa correlacionaram-se significativamente com a dimensão ciências exatas, o que é coerente com as definições dos autores (Noronha et al., 2007; Holland, 1975). Os autores reafirmaram as relações entre a análise e a crítica do tipo investigativo com a habilidade numérica da dimensão ciências exatas e a praticidade e a objetividade do realista, características previstas na carreira de Engenharia.

A dimensão artes e comunicação correlacionou-se positivamente com o tipo artístico, o que indica coerência na combinação do caráter criativo, imaginativo e intuitivo. As tipologias investigativo e social, normalmente apresentadas por sujeitos que buscam ocupações na área das ciências da saúde e pesquisa e preocupação com o outro, apareceram associadas expressivamente com a dimensão ciências biológicas, que representa tais ocupações na EAP. Já o tipo investigativo apresentou correlação com a dimensão ciências agrárias e ambientais, também corroborando os achados de Noronha e Ambiel (2008).

A dimensão atividades burocráticas, ainda, correlacionou-se positivamente com a tipologia convencional, empreendedor, investigativo e realista. A dimensão atividades humanas e sociais, apresentaram correlações significativas com os tipos artístico, convencional, empreendedor e 
social. Esses achados podem, parcialmente, corroborar os resultados de Nauta, nos quais foram encontradas associações entre o tipo artístico do SDS e a socialização dos cinco grandes fatores.

Por fim, a dimensão entretenimento do EAP correlacionou-se positivamente com o tipo empreendedor do ATPH, o que parece coerente, uma vez que, no EAP, essa dimensão é composta por atividades de gerenciamento (Noronha et al., 2007), o que está em consonância com as características dominante, impulsivo e de convencimento do tipo empreendedor (Holland, 1975).

\section{Considerações finais}

O estudo de evidências de validade convergente-discriminante do ATPH, por meio de comparação com a EAP, revelou que há associações entre os instrumentos. Dessa forma, pode-se inferir que os conceitos de interesses adotados pelos respectivos autores, embora se diferenciem em um ou outro aspecto, convergem para uma compreensão comum.
Neste estudo, foram realizadas duas análises, comparação de média entre sexos e correlação entre as dimensões dos instrumentos. No que respeita à primeira análise, não foram encontradas diferenças de médias em nenhuma das dimensões da EAP. Já nas dimensões do ATPH, foram encontradas médias mais altas no tipo social, para o sexo feminino, e no tipo realista, para os homens. As correlações entre as dimensões dos instrumentos mostraram-se significativas, o que indica a comunalidade entre os construtos.

Pode-se afirmar, a partir do exposto, que o ATPH apresentou validade, ou seja, ele possui evidências de que avalia interesses profissionais. Tendo em vista que este foi o primeiro dos estudos realizados com esse intuito, outras investigações são necessárias, a fim de sua aplicação possa ser indicada para utilização em situações individuais ou coletivas em processos de orientação profissional. A comparação com outros construtos, com instrumentos diversos, e a ampliação da amostra são algumas das sugestões para pesquisas futuras. 


\section{Ana Paula Porto Noronha}

Doutora em Psicologia, Ciência e Profissão pela Pontifícia Universidade Católica de Campinas e docente do programa de pós-graduação Stricto Sensu em Psicologia da Universidade São Francisco, Itatiba - SP - Brasil.

E-mail: ananoronha@saofrancisco.edu.br

\section{Camélia Santina Murgo Mansão}

Doutora em Psicologia, Ciência e Profissão pela Pontifícia Universidade Católica de Campinas,

Campinas - SP - Brasil.

E-mail: murgo@netsite.com.br

\section{Marlene Alves da Silva}

Doutoranda do Programa de pós-graduação Stricto Sensu em Psicologia da Universidade São Francisco e docente substituta da Universidade Federal da Bahia,

Vitória da Conquista - BA - Brasil.

E-mail: alvesmarlene2002@yahoo.com.br

\section{Patrícia Carla dos Santos Freitas}

Psicóloga formada pela Universidade São Francisco,

Itatiba - SP - Brasil.

E-mail:pfc_psico@yahho.com.br"

\section{Geruza Oliveira Aquino Pereira}

Psicóloga formada pela Universidade São Francisco,

Itatiba - SP - Brasil.

E-mail: psico.geruza_oliveira@hotmail.com"

\section{Endereço para envio de correspondência:}

Avenida Otávio Santos, 261, sala 08, Centro. CEP: 4520-070. Vitória da Conquista, BA 
Crites, J. O. (1969). Vocational psychology. New York: MacgrawHill.

Holland, J. L. (1975). Técnica de la elección vocacional: Tipos de personality modelos ambientales (R. D. Guerrero, trad.). México: Trillas.

Holland, J. L., Fritzshe, B. A., \& Powell, A. B. (1994). SDS SelfDirected Search Technical Manual. Flórida: Psychological Assessment Resources, Inc.

Mansão, C. S. M., \& Yoshida, E. P. M. (2008). SDS - Questionário de Busca Auto-Dirigida: precisão e validade. Revista Brasileira de Orientação Profissional, 7(2), 67-79.

Nauta, M. M. (2007). Career interests, self-efficacy, and personality as antecedents of career exploration. Journal of Career Assessment, 15(2), 162-180.

Noronha, A. P., Sisto, F., \& Santos, A. A. A. (2007). Escala de Aconselhamento Profissional (EAP) - Manual Técnico (Brasil). São Paulo: Vetor.

Noronha, A. P. P., \& Ambiel, R. A. M. (2008). Estudo correlacional entre Escala de Aconselhamento Profissional (EAP) e Selfdirected Search (SDS). Interação, 12, 21-33.

Noronha, A. P. P., Martins, D. F., Gurgel, M. G. A., \& Ambiel, R. A. M. (2009). Estudo correlacional entre interesses profissionais e vivências acadêmicas. Psicologia Escolar e Educacional, $13,143-154$

Noronha, A. P. P., \& Nunes, M. F. O. (2009). Interesses e personalidade: um estudo com adolescentes em orientação profissional. Revista Galego-Portuguesa de Psicoloxía e Educación, 17,115-139.
Primi, R., Pellegrini, M. C. K., Nucci, E. P., Munhoz, A . H., Bighetti, C. A., \& Moggi, M. A. (2001). Características de personalidade e indecisão profissional. Psico, 1, 82-96.

Primi, R., Moggi, M. A., \& Casellato, E. O. (2004). Estudo correlacional do Inventário de Busca Autodirigida (Self-Directed Search) com o IFP. Psicologia Escolar e Educacional, 8(1), 47-54.

Primi, R. Muniz, M. Nunes, M., \& Murgo, C. M. (2008). Avaliação dos tipos profissionais de Holland - ATPH. (Relatório técnico não publicado).

Sartori, F. A., Noronha, A. P. P., \& Nunes, M. F. O. (2009). Comparações entre EAP e SDS: interesses profissionais em alunos do ensino médio. Boletim de Psicologia, LIX 130,17-29.

Savickas, M. L. (1995). Examining the personal meaning of inventoried interests during career counseling. Journal of Career Assessment, 3(2),188-201.

Savickas, M. L. (1999). The psychology of interests. In M. L. Savickas \& A. R. Spokane (Eds.), Vocational interests. Meanings, measurement and counseling use (pp. 19-56). Palo Alto, CA: Davies-Black.

Sisto, F. F., Santos, A. A. A., \& Noronha, A. P. P. (2009). Escala de Aconselhamento Profissional: carreira universitária como evidência de validade de critério. Revista Galego-Portuguesa de Psicoloxia e Educación, 17(1/2), 1138-1663.

Super, D. E. (1953). A theory of vocational development. American Psychologist, 8, 185-190. 